\title{
ARTÍCULO ORIGINAL \\ Concordancia entre el diagnóstico clínico presuntivo y el diagnóstico tomográfico abdomino-pélvico
} \author{
Muñoz Bendezú, Zunilda Leyda ${ }^{4}$

\begin{abstract}
Médico Hospital Augusto Hernández Mendoza - EsSalud Ica1
Médico Natural and Social Sciences Research2

Licenciada en Enfermería Natural and Social Sciences Research3

Médico Hospital Augusto Hernández Mendoza - EsSalud Ica4
\end{abstract}

Goicochea Ruiz, Virginia Nelly ${ }^{1}$, Oyola García, Alfredo Enrique ${ }^{2}$, Quispe Ilanzo, Melisa Pamela ${ }^{3}$

\section{RESUMEN}

Objetivo: Determinar la correlación entre el diagnóstico clínico presuntivo y el diagnóstico tomográfico abdomino-pélvico en el servicio de Imagenología del Hospital Augusto Hernández Mendoza - EsSalud, Ica durante el año 2013. Materiales y métodos: Se realizó un estudio observacional, descriptivo y retrospectivo, seleccionando 599 registros de estudio tomográfico abdominal y pélvico de pacientes de 18 años a más, atendidos en el servicio de Imagenología del Hospital Augusto Hernández Mendoza EsSalud, Ica de enero a diciembre 2013; excluyéndose 252 (42,07\%) registros que no tenían resultado del estudio y cinco de menores de 18 años por lo que la muestra final quedó conformada por 342 registros Resultados: $32,16 \%$ de las solicitudes provienen de la especialidad de medicina interna. $61,11 \%$ de los pacientes evaluados fueron de sexo femenino, $68,53 \%$ tenían 50 años o más, $52,45 \%$ correspondía al grupo etario de 60 a más años, asimismo, $42,50 \%$ pertenecía al grupo de 30 a 59 años. $80,70 \%$ de los exámenes correspondieron a tomografías helicoidales de abdomen con contraste. En los resultados tomográficos $36,27 \%$ tenía afectación gastrointestinal y/o hepática, 21,69\% urinaria y 16,27\% oncológica. El resultado tomográfico fue concordante con el diagnóstico presuntivo en 103 (34,92\%) registros. La correlación no se asoció a ninguna de las variables evaluadas. En 47 (13,7\%) de los registros no se había consignado el diagnóstico presuntivo. Conclusión: Existe elevada frecuencia de discordancia entre el resultado tomográfico y el diagnóstico presuntivo. Uno de cada diez exámenes no cuenta con diagnóstico presuntivo. Palabras clave: Diagnóstico; tomografía; Abdomen. (Fuente: DeCS BIREME). Autor corresponsal: Goicochea Ruiz, Virginia Nelly, Teléfono: (51) 988062421. Correo electrónico: vickyngoru@hotmail.com

\section{INTRODUCCIÓN}

De todos los pacientes que acuden al servicio de emergencias uno de cada diez refiere como motivo de consulta el dolor abdominal agudo. Este puede ser causado por una amplia variedad de condiciones.

El diagnóstico por imágenes es ampliamente utilizado en el trabajo de los pacientes con dolor abdominal agudo. La tomografía computarizada es usada con frecuencia como ayuda diagnóstica principal de la evaluación clínica y de laboratorio(2). Es un procedimiento de diagnóstico por imágenes que utiliza una combinación de rayos $X y$ tecnología computarizada para obtener imágenes horizontales o transversales (a menudo llamadas cortes) del cuerpo $(3,4)$. Suelen proporcionar más detalles que las radiografías tradicionales, sobre todo de los tejidos blandos y vasos sanguíneos(3).

Las tomografías computarizadas pueden realizarse con o sin "contraste", para lograr que el órgano o tejido en particular que se está estudiando se vea con más claridad(4).
La tomografía helicoidal multicorte es una de las técnicas modernas y avanzadas que han revolucionado los métodos tradicionales de ayuda diagnóstica(2,5,6,7). Se introdujo por primera vez como un dispositivo médico en la década de 1970(6,7). En 1977, revolucionó la neurorradiología y su impacto en la imagen abdominal y pélvica había sido igualmente grande ${ }^{6}$

Para Pothiawala y Gogna (Singapur, 2012)(8) la tomografía computarizada de abdomen de emergencia sirve como una herramienta de diagnóstico importante, en vista de su capacidad para detectar el sitio, el nivel y la causa de la obstrucción intestinal, junto con el aspecto distintivo de obstrucción de intestino delgado y los signos de isquemia. Díaz-Sánchez et al. (México, 2011)(9), señalan que la tomografía tiene alta sensibilidad y especificidad para el diagnóstico de la apendicitis aguda, así como para identificar complicaciones de la misma y facilitar el pronto manejo de los pacientes disminuyendo la morbimortalidad. Van Randem et al. (Europa, 2011)(10), observaron que la sensibilidad de la tomografía computarizada en la detección de la apendicitis y la diverticulitis fue significativamente mayor que la de ultrasonido: $94 \%$ frente a $76 \%(p<0,01)$ y $81 \%$ frente a $61 \%(p=0,048)$, respectivamente. De igual forma, Hlibczuk et al. (EE.UU., 2010)(11), mediante una búsqueda en MEDLINE, EMBASE, The Cochrane Library y las bibliografías de las revisiones sistemáticas hallaron que la exactitud diagnóstica de la tomografía computarizada sin contraste para el diagnóstico de apendicitis aguda en la población adulta es adecuada para la toma de decisiones clínicas en el servicio de emergencias. Lameris et al. (Holanda, 2009)(2), mediante un estudio multicéntrico prospectivo en los servicios de urgencias de dos hospitales universitarios y cuatro grandes hospitales de enseñanza en los Países Bajos, observaron que, aunque la tomografía computarizada es la prueba de imagen más sensible para la detección de condiciones de urgencia en pacientes con dolor abdominal, el uso de la ecografía en primer lugar y luego la tomografía computarizada -sólo en aquellos con resultados negativos o no concluyentesgenera mejor sensibilidad y reduce la exposición a la radiación. Fraser et al (Kansas, 2009)(12), en una investigación realizada con el objetivo de cuantificar la capacidad de la tomografía computarizada para identificar qué pacientes cumplen con criterios de perforación apendicular, halló $72 \%$ de concordancia de los casos, con una sensibilidad de $62 \%$ y una especificidad del $81 \%$. El valor predictivo positivo fue $67 \%$ y el valor predictivo negativo $77 \%$.

Sin embargo, con el transcurrir de los años, el diagnóstico por imagen en el departamento de emergencia ha sido responsable de un aumento en los costos hospitalarios $(13,14)$, y la tomografía computarizada ha sido una creciente fuente de exposición a la radiación en pacientes adultos(15). La exposición a la radiación ionizante en la tomografía ha sido 
asociada con el riesgo de cáncer inducido por radiación. Más de dos tercios de toda la radiación médica es atribuible a la tomografía computarizada, $75 \%$ de ellas son realizadas en el ámbito hospitalario y $47 \%$ son del abdomen/pelvis $(7,15,16)$. Este es un inconveniente de la tomografía computarizada, sobre todo porque la tomografía se utiliza cada vez más en el estudio diagnóstico de pacientes jóvenes(10).

En el Servicio de Imagenología del Hospital Augusto Hernández Mendoza - EsSalud se realizan miles de estas pruebas al año, solicitadas por referencia de diferentes servicios y en la que se obtienen variados diagnósticos. Sin embargo, a pesar de ser una prueba diagnóstica que tiene un papel esencial en la evaluación de pacientes, en nuestro medio aún no se ha evaluado de manera objetiva la concordancia entre el motivo de la solicitud -es decir, la sospecha clínica del facultativo- y el diagnóstico de patologías abdominales y/o pélvicas. Asimismo y, como hemos expuesto, la TEM se presenta como una importante ayuda diagnóstica, sin embargo, su uso indiscriminado puede tener consecuencias sobre el paciente. En tal sentido, planteamos el presente estudio con el objetivo de determinar la correlación entre el diagnóstico clínico presuntivo y el diagnóstico tomográfico abdomino-pélvico en el servicio de Imagenología del Hospital Augusto Hernández Mendoza - EsSalud, Ica durante el año 2013.

\section{MATERIALES Y MÉTODOS}

Se realizó un estudio observacional, descriptivo y retrospectivo, en una población total 2980 registros de pacientes sometidos a estudio tomográfico helicoidal abdominal y/o pélvico en el Servicio de Imagenología del Hospital Augusto Hernández Mendoza - EsSalud, Ica durante el año 2013. La muestra se determinó al máximo poder muestral usando la fórmula para población conocida: $n=[N p(1-p)] /$ $[(d 2 / Z 21-\alpha / 2 *(N-1)+p *(1-p)] ;$ donde: población $(N)=547$; significancia $(Z)$ al $95 \%=$ 1,96; proporción del problema $(p)=0,5 ; q$ (1$p)=0,5$; error $(E)=0,05$. Con estos datos la muestra calculada ascendió a 341 registros de pacientes, seleccionados mediante muestreo aleatorio simple utilizando los códigos de registro de los exámenes tomográficos realizados y la función números aleatorios del programa MS EXCEL ${ }^{\circledR}$ para Windows.

Se seleccionaron 599 registros estudio tomográfico abdominal y pélvico de pacientes de 18 años a más, atendidos en el servicio de Imagenología del Hospital
Augusto Hernández Mendoza - EsSalud, Ica entre enero y diciembre del 2013 , cuya ficha de registro tomográfico se encontraba en el archivo del hospital; excluyéndose 252 $(42,07 \%)$ registros que no tenían resultado del estudio y cinco registros de pacientes menores de 18 años por lo que la muestra final quedó conformada por 342 registros.

La recolección de datos se hizo mediante la ficha de recolección diseñada para tal efecto. En esta ficha se incluyeron las variables: diagnóstico imagenológico mediante tomografía abdominal y pélvica, diagnóstico presuntivo, edad, sexo, servicio solicitante, tipo de tomografía, especialidad del médico solicitante (Anexo 1).

La base datos se construyó usando el programa Statistical Package for Social Sciences (SPSS) versión 21.0. Los datos de las fichas de observación fueron digitados previo control de calidad para verificar que todas las variables estén completadas. Culminada esta fase se llevó a cabo un segundo control de calidad para eliminar las inconsistencias en los datos de las variables. Luego, se aplicó la prueba de Chi cuadrado para determinar la asociación de la variable dependiente con las variables independientes, aceptando la relación estadísticamente significativa si el valor $p$ es menor de 0,05.

El presente estudio al no ser un estudio experimental sino descriptivo no sometió a riesgo la integridad de ninguno de los pacientes. Para mantener la confidencialidad de los datos personales de los pacientes, no se colocó el nombre de ellos en la base de datos del trabajo de investigación, sólo se registró el número de ficha correspondiente de acuerdo al registro del servicio de Imagenología. Con este último dato se pudo cotejar la información registrada en caso fue necesario. La confidencialidad de los datos fue preservada, garantizando que la administración de los datos estuviera a cargo de la investigadora. El estudio fue aprobado por la Facultad de Medicina de la Universidad Nacional San Luis Gonzaga y, su ejecución, autorizada por el Director del nosocomio.

\section{RESULTADOS}

En relación a la procedencia del paciente, 290 (84,80\%) provenían del hospital "Augusto Hernández Mendoza", 26 (7,60\%) del hospital "Félix Torrealva Gutiérrez", 16 (4,68\%) del hospital "Antonio Skrabonja Antonich", 9 (2,63\%) del hospital "René Toche Groppo" y uno (0,29\%) del hospital María Reiche Newmann (Tabla 1).
De los pacientes pertenecientes al Hospital Augusto Hernández Mendoza (290), 178 (61,38\%) provenían de consultorio externo, $88(30,34 \%)$ de emergencia, $24(8,28 \%)$ de hospitalización (Tabla 1).

Medicina Interna, Oncología y Cirugía General son los servicios que demandan el mayor número de exámenes tomográficos. En la muestra estudiada 110 (32,07\%) fueron solicitados por el Servicio de Medicina, 42 $(12,24 \%)$ por el servicio de Oncología y 36 $(10,50 \%)$ por Cirugía General (Tabla 1).

En relación con el sexo, se pudo observar que $209(61,11 \%)$ de los pacientes eran de sexo femenino y $133(38,89 \%)$ de sexo masculino (Tabla 1).

$68,53 \%$ de los pacientes que fueron sometidos a este examen tenían 50 años o más. $52,45 \%$ de ellos correspondía al grupo etario de 60 a más años. Asimismo, 42,50\% pertenecía al grupo de 30 a 59 años (Gráfico 1).

$276(80,70 \%)$ de los pacientes fueron sometidos a tomografía helicoidal de abdomen con contraste, $34(9,94 \%)$ a tomografía helicoidal abdomino-pélvica con contraste, mientras que la tomografía helicoidal de abdomen sin contraste fue realizada en $28(7,8 \%)$ pacientes. Los demás tipos de tomografía se registraron en menos del $5 \%$ (Tabla 2).

Según los diagnósticos tomográficos, en 107 $(36,27 \%)$ de los casos hubo afectación gastrointestinal y/o hepática, en 64 (21,69\%) esta fue urinaria y en $48(16,27 \%)$ oncológica. La afectación ginecológica se observó en 17 (5,76\%) casos. La afectación de otros aparatos o sistemas se observó en menos del $5 \%$ (Gráfico 2).

En 47 (13,70\%) de los registros no se había consignado el diagnóstico presuntivo. De los 295 registros de pacientes con diagnóstico presuntivo, se observó que $103(34,92 \%)$ de estos diagnósticos fueron concordantes con el diagnóstico tomográfico. En 192 (65,08\%) estos no fueron concordantes (Tabla 3).

La correlación fue inferior al $50 \%$ en todos los hospitales solicitantes. $36,21 \% ; 26,92 \%$ y $12,50 \%$ fueron los porcentajes de concordancia de los hospitales Augusto Hernández Mendoza, Félix Torrealva Guitiérrez y Antonio Skrabonja Antonich, respectivamente. Sin embargo, los casos procedentes de otros hospitales mostraron mayor porcentaje de correlación (60\%). La correlación no se asoció al hospital de procedencia. 
Tabla 1:

EXAMENES TOMOGRÁFICOS ABDOMINO-PÉLVICOS

SEGÚN CARACTERÍSTICAS GENERALES DEL PACIENTE. HOSPITAL AUGUSTO HERNÁNDEZ MENDOZA - ESSALUD, ICA. 2013

\begin{tabular}{|c|c|c|}
\hline CARACTERÍSTICAS GENERALES DEL PACIENTE & $\mathbf{n}$ & $\%$ \\
\hline Sexo del paciente & & \\
\hline Femenino & 209 & 61,11 \\
\hline Masculino & 133 & 38,89 \\
\hline Procedencia & & \\
\hline Hosp. Augusto Hernández Mendoza & 290 & 84,8 \\
\hline Hosp Félix Torrealva Gutiérrez & 26 & 7,6 \\
\hline Hosp Antonio Skrabonja Antonich & 16 & 4,68 \\
\hline Hosp René Toche Groppo & 9 & 2,63 \\
\hline Hosp María Reiche Newmann & 1 & 0,29 \\
\hline Area solicitante & & \\
\hline Consultorio Externo & 178 & 61,38 \\
\hline Emergencia & 88 & 30,34 \\
\hline Hospitalizacion & 24 & 8,28 \\
\hline Medicina Interna & 110 & 32,16 \\
\hline Oncologia & 42 & 12,28 \\
\hline Cirugia General & 290 & 84.8 \\
\hline Urologia & 36 & 10,53 \\
\hline Gastroenterología & 34 & 9,94 \\
\hline Nefrología & 30 & 8,77 \\
\hline Ginecología y Obstetricia & 27 & 7,89 \\
\hline Hemodiálisis & 8 & 2,34 \\
\hline Neumología & 4 & 1,17 \\
\hline Endocrinología & 4 & 1,17 \\
\hline Cardiología & 3 & 0,88 \\
\hline Cirugía Plástica, Quemados y Reconstructiva & 2 & 0,58 \\
\hline Otros & 2 & 0,58 \\
\hline Sin dato & 32 & 9,36 \\
\hline
\end{tabular}

solicitante.

De igual forma la frecuencia de aciertos según el tipo de tomografía helicoidal realizada fue inferior al 50\%. Los resultados no concordantes fueron más frecuentes cuando se realizaron tomografías helicoidales de abdomen sin contraste (17 casos; $77,27 \%)$. La correlación no se asoció al tipo de tomografía helicoidal realizada.

Cuando en el resultado tomográfico hubo afectación de tipo oncológica o ginecológica, hubo mayor frecuencia de correlación; 23 $(47,92 \%)$ y $8(47,06 \%)$ concordancias, respectivamente. La más baja frecuencia de aciertos se observó cuando el resultado fue normal $(8 ; 19,51 \%)$, mientras que -excluyendo estos resultados normales- la mayor frecuencia de desaciertos se observó cuando la afectación fue gastrointestinal y/o hepática $(74 ; 69,16 \%)$.

\section{DISCUSION}

La labor del clínico frente a un enfermo que acude con una molestia es en averiguar el origen de sus molestias (juicio diagnóstico), luego valorar las consecuencias de ese mal para la vida y el bienestar del paciente (juicio pronóstico) y, finalmente, intentar resolver o mitigar el problema (indicación terapéutica)(17)

En este contexto el diagnóstico por imágenes juega un rol importante en la práctica médica para la confirmación o descarte del diagnóstico. Existen diferentes modalidades diagnósticas que van desde la simple radiografía hasta la resonancia magnética nuclear pasando por la tomografía axial computada (helicoidal - multicorte), el ecodoppler, entre otros. De estos, la tomografía helicoidal es un método
La correlación fue inferior al 50\% en todos los servicios solicitantes. Sin embargo, en los casos procedentes del servicio de emergencia y de hospitalización se observó mayor porcentaje de correlación: 19 $(46,34 \%)$ y $10(41,67 \%)$ casos, respectivamente. La frecuencia más alta de discordancia se observó en los casos provenientes de consultorio externo (119, $66,85 \%)$. La correlación no se asoció al servicio solicitante.

Todas las especialidades tuvieron una frecuencia de acierto inferior al 50\%. La frecuencia más baja de correlación se observó en la especialidad de Oncología (8 concordancias; 19,05\%). Cirugía General y Nefrología fueron las especialidades con mayor frecuencia de aciertos; 16 (44,44\%) y $12(44,44 \%)$, respectivamente. La correlación no se asoció a la especialidad
Gráfico 1:

EXAMENES TOMOGRÁFICOS ABDOMINO-PÉLVICOS SEGÚN EDAD DEL PACIENTE.

HOSPITAL AUGUSTO HERNÁNDEZ MENDOZA - ESSALUD, ICA. 2013

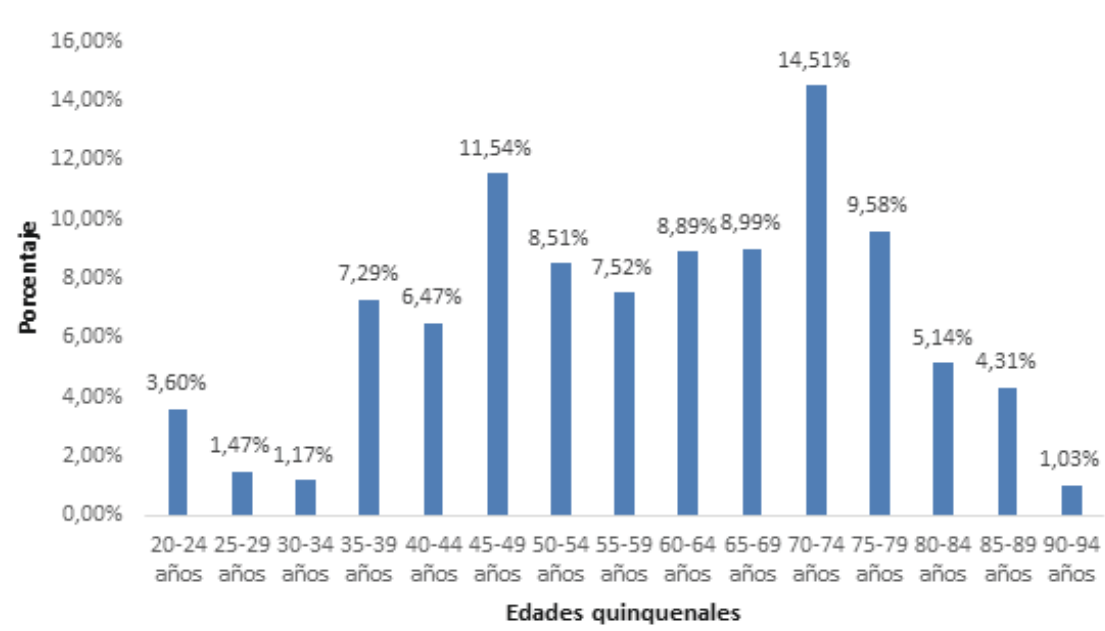


de diagnóstico cuyo su uso se ha hecho frecuente en los establecimientos de salud de mediana y alta complejidad(18). Sin embargo, esta creciente demanda podría estar dejando de lado una adecuada evaluación y juicio clínicos que a la larga generan mayor costo en la atención de pacientes, por el uso no fundamentado de este examen, pues entre nuestros resultados se observa que uno de cada diez exámenes realizados no tiene un diagnóstico presuntivo. Los exámenes de ayuda diagnóstica, como la tomografía helicoidal, tienen por función ayudar a la confirmación del diagnóstico clínico(17,19).

El porcentaje de pacientes que no presentan anormalidades evidenciables a la luz de este examen es bajo, solo uno de cada diez de los pacientes evaluados. Sin embargo, existe elevado porcentaje de discordancia entre los hallazgos tomográficos y el diagnóstico presuntivo, principalmente cuando estos provienen del área de consulta externa. Esto nos indica que la tomografía helicoidal abdomino-pélvica se usa como un examen exploratorio y no como un método de ayuda para la confirmación de la presunción que el clínico ha establecido mediante un exhaustivo examen como lo precisan

Tabla 2:

EXAMENES TOMOGRÁFICOS ABDOMINO-PÉLVICOS SEGÚN TIPO DE TOMORAFIA HELICOIDAL REALIZADA. HOSPITAL AUGUSTO HERNÁNDEZ MENDOZA - ESSALUD, ICA. 2013

\begin{tabular}{lrr}
\hline TIPO DE TOMORAFíA HELICOIDAL & n & \multicolumn{1}{c}{$\%$} \\
\hline Abdomen con contraste & 276 & 80,70 \\
Abdomino-pélvica con contraste & 34 & 9,94 \\
Abdomen sin contraste & 28 & 7,89 \\
Abdomen con contraste (trifásico-dinámico) & 3 & 0,88 \\
Abdomino-pélvica sin contraste & 2 & 0,58
\end{tabular}

Arteaga y Fernández(19). Noriega-Negrete y Guerrero-Avendaño(20) también han encontrado similares resultados, pues observaron que concordancia entre el diagnóstico presuntivo de litiasis renal y el resultado tomográfico fue $22,5 \%$; además, en $51 \%$ de los casos no se detectó patología urinaria alguna, a pesar de tener un diagnóstico presuntivo de litiasis urinaria.

Esta característica es mucho más frecuente cuando la solicitud de examen proviene de otros hospitales, la especialidad que lo solicita es Oncología o cuando la afectación que se evidencia a través de este examen es gastrointestinal o hepática. Aunque hemos esgrimido que una de las condicionantes de esta baja concordancia es la agudeza clínica, Pothiawala y Gogna(8), han demostrado que este examen es crucial para la identificación de ciertas afecciones gastrointestinales, con

$n=295$

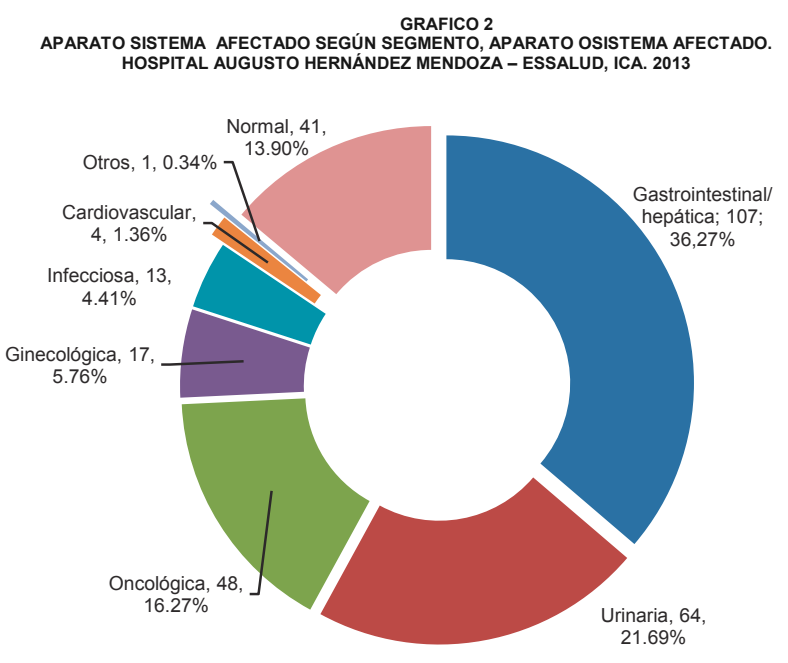

elevada sensibilidad y especificidad en algunas de ellas, como lo demuestra Díaz Sánchez et al(9) y mayor a la reportada en la ecografía, según los estudios de Van Randem et al(10).

Adicionalmente, durante la ejecución del estudio, se pudo evidenciar que más de la tercera parte de los exámenes tomográficos no contaba con resultados de los hallazgos observados.

Situación que evidencia deficiencias en el monitoreo y evaluación de los procesos que se instalan para la atención del paciente. Asimismo, un examen que no cuenta con informe de los hallazgos podría considerarse como un examen sin importancia para la evaluación del paciente que ocasiona la exposición innecesaria de este a riesgos derivados de la radiación, así como el gasto de recursos económicos y humanos.

Estos últimos por la sobrecarga laboral que también genera exposición innecesaria a radiación. La exposición a la radiación de la tomografía computarizada es más alta que la de procedimientos regulares de rayos $X(21)$.

Una importante limitación para la realización de este estudio ha sido la escasa información disponible sobre el tema, pues existen muy pocos estudios que valoren la concordancia del diagnóstico clínico con los resultados de los exámenes de ayuda diagnóstica, en nuestro caso, la tomografía helicoidal computarizada.

Sin embargo, al no existir estudios previos en nuestro medio, nos permitió conocer la distribución de diagnósticos y demanda de la tomografía helicoidal abdominal y pélvica. Asimismo, el conocimiento de esta información permitirá mejorar la evaluación y referencia de los pacientes, y así evitar solicitudes y tiempos de espera innecesarios. Los resultados de esta investigación servirán para mejorar la forma y la frecuencia en que se realizan las solicitudes de examen al servicio de Imagenología.

Concluimos que, el acierto diagnóstico, es decir, la correlación o concordancia se observa solo en uno de cada tres pacientes evaluados. Además, uno de cada diez pacientes no cuenta con diagnóstico presuntivo.

TABLA 3:

PORCENTAJE DE CORRELACIÓN ENTRE EL DIAGNÓSTICO CLÍNICO PRESUNTIVO Y EL DIAGNÓSTICO TOMOGRÁFICO ABDOMINO-PÉLVICO. HOSPITAL AUGUSTO HERNÁNDEZ MENDOZA - ESSALUD, ICA. 2013

\begin{tabular}{lll}
\multicolumn{1}{c}{ CORRELACION } & $\mathbf{n}$ & $\mathbf{\%}$ \\
No hubo correlación & 192 & 65,08 \\
Si hubo correlación & 103 & 34,92 \\
\hline
\end{tabular}


$\mathrm{F} \quad$ əbemos precisar que el diagnostico clinico presuntivo es una hipótesis con una certidumbre más o menos intensa y con un grado de corroboración más o menos grande en la que debemos basar nuestra actuación. Este debe surgir del razonamiento clínico gracias a los datos que se obtienen del interrogatorio y de la exploración física, además de las pruebas complementarias(17). No se basa, exclusivamente, en los exámenes complementarios. Por esta razón es importante establecer y fomentar la discusión de casos clínicos que evalúen la pertinencia del uso de la tomografía helicoidal como método de ayuda diagnóstica; promover en los médicos asistentes el uso del método clínico durante la atención del paciente y su registro en la historia clínica y los actos médicos realizados, con énfasis en el personal que que labora en consultorio externo; así como implementar un plan de monitoreo y evaluación de los procesos relacionados con el uso de exámenes de ayuda diagnóstica.

\section{REFERENCIAS BIBLIOGRÁFICAS}

1.Stoker J, Randen A, Laméris $W$, Boermeester MA. Imaging patients with acute abdominal pain. Radiology. 2009; 253:31-46.

2. Lameris $W$ et al. Imaging strategies for detection of urgent conditions in patients with acute abdominal pain: diagnostic accuracy study. BMJ (internet). 2009 (citado 19 ago 14). 338:b2431. Disponible en:

http://www.bmj.com/content/338/bmj. b2431.long

3. Computed Tomography (CT) - Abdomen and Pelvis. 2014 (internet). Radiologyinfo.org. (citado 05 mar 14) D i s o n i b e e n : http://www.radiologyinfo.org/en/info.cf m?pg=abdominct

4. Johns Hopkins Medicine. Computed Tomography (CT or CAT) Scan of the Abdomen. Health Library (internet) (citado 05 mar 14) Disponible en: http://www.hopkinsmedicine.org/health library/test_procedures/gastroenterolog y/computed_tomography_ct_or_cat_sca $\mathrm{n}$ of the abdomen 92,P07690/

5. Miguel-Pérez I, Domínguez-Expósito MG, García-Ferrer LU. La tomografía computarizada helicoidal versus ecografía en el diagnóstico de un cuerpo extraño intraocular (internet). Revista Cubana de Oftalmología. (citado 16 may 14). Disponible en:

http://bvs.sld.cu/revistas/oft/vol18 10 5/oft08105.pdf

6. Sluimer I, Schilham A, Prokop M, van Ginneken B. computer analysis of computed tomography scans of the lung: a survey. IEEE Transactions on Medical Imaging. 2006; 25(4):385-405.

7. Guite KM, Hinshaw JL, Lee FT. Computed tomography in abdominal imaging: how to gain maximum diagnostic information at the lowest radiation dose. En: Wang D, Ed. Selected Topics on Computed Tomography (internet). 2013. (citado 16 may 14) Disponible en: http://www.intechopen.com/books/sele c t e d - to p i c s - on - com p u t ed tomography/computed-tomography-inabdominal-imaging-how-to-gainmaximum-diagnostic-information-atthe-lowest-ra

8. Pothiawala S, Gogna A. Early diagnosis of bowel obstruction and strangulation by computed tomography in emergency department. World J Emerg Med 2012;3(3):227-231.

9. Díaz-Sánchez ME, Castillo JO, Treviño-Frutos RJ. Eficacia de la tomografía computada en el estudio de apendicitis aguda; correlación anatomopatológica. Anales de Radiología México 2011;3:194-199

10. Van Randen A et al. A comparison of the accuracy of ultrasound and computed tomography in common diagnoses causing acute abdominal pain. Eur Radiol (internet). 2011 (citado 19 ago 14). 21:1535-545. Disponible en: http://download.springer.com/static/pd f/278/art\%253A10.1007\%252Fs00330011-2087-5.pdf?auth66=1413728492_5 345f5b2d97817504e6bc95716ac6bb5\& ext=.pdf

11. Hlibczuk V, Dattaro JA, Jin Z, Falzon L, Brown MD. Diagnostic accuracy of noncontrast computed tomography for appendicitis in adults: a systematic review. Annals of Emergency Medicine (internet). 2010 (citado 19 ago 14); 55(1):51-9.e1. Disponible en: http://www.annemergmed.com/article /S0196-0644(09)01140-8/abstract

12. Fraser JD et al. Accuracy of computed tomography in predicting appendiceal perforation. Journal of Pediatric Surgery (2010) 45, 231-235.

13.Broder J, Warshauer DM. Increasing utilization of computed tomography in the adult emergency department, 20002005. Emerg Radiol 2006; 13:25-30.

14. Mitka M. Costly surge in diagnostic imaging spurs debate. JAMA 2005; 293:665-7.

15.Brenner, DJ, Hall EJ.Computed Tomography - An Increasing Source of Radiation Exposure. NEJM, 2007;357:2277-2284

16. Brix G, Nissen-Meyer S, Lechel U, et al. Radiation exposures of cancer patients from medical x-rays: how relevant are they for individual patients and population exposure? European Journal of Radiology, 2009;72(2):342-347.

17.Sangrós FJ, Astier MP. Utilización adecuada de las pruebas diagnósticas en su contexto epidemiológico: la prevalencia en el escenario clínico como parte del proceso diagnóstico. Revista clínica electrónica en atención primaria. 2009; 17:1-6.

18. Quezada-Sanhueza N, León-Ferrufino F, Bächler-González J, Riquelme-Pizarro C, Crovari-Eulufi F, Jarufe-Cassis N. Rol de la tomografía computada de abdomen y pelvis con contraste intravenoso en las decisiones clínicas de pacientes con obstrucción de intestino delgado por bridas. Cir Cir 2014;82:637-646.

19. Arteaga-Herrera J, Fernández-Sacasas JA. El método clínico y el método científico. Medisur 2010; 8(5):12-20.

20. Noriega-Negrete I, Guerrero-Avendaño G. Hallazgos tomográficos en pacientes con sospecha clínica de urolitiasis. Evaluación de la certeza clínica y las afecciones asociadas más frecuentes. Anales de Radiología México 2013;1:2-6

21. Raslawski EC. Tomografía computada. Una fuente considerable de exposición a la radiación. Arch. argent. pediatr. 2008; 106(3):273-274 
FINANCIAMIENTO: Autofinanciado

CONTRIBUCIONES DE AUTORÍA: GRV, OGG, QLLM y MBZ

participaron en el diseño del estudio, el análisis de los datos,

revisaron críticamente el artículo y aprobaron la versión final.

\section{Concordancia entre el diagnóstico clínico presuntivo y el diagnóstico tomográfico abdomino-pélvico}

Objective: To determine the correlation between the presumptive clinical diagnosis and abdomino-pelvic CT diagnosis at the Imaging service of Augusto Hernández Mendoza Hospital - EsSalud, Ica during 2013. Materials and Methods: An observational, descriptive and retrospective study was conducted, selecting 599 abdominal and pelvic tomographic study records of patients 18 years or older, treated in the Imaging of Augusto Hernández Mendoza Hospital - EsSalud, Ica from January to December 2013; excluding 252 (42.07\%) records that did not have result from the study and five under 18 years old, so the final sample was composed of 342 records. Results: $32.16 \%$ of the requests come from the specialty of internal medicine. $61.1 \%$ of the evaluated patients were female, $68.53 \%$ were 50 years or older, $52.45 \%$ belonged to the age group 60 years and older, also $42.50 \%$ belonged to the group of $30-59$ years. $80.70 \%$ of the reviews were for helical CT with contrast of the abdomen. In the tomographic findings $36.27 \%$ had gastrointestinal and/or liver involvement, $21.69 \%$ urinary one and $16.27 \%$ oncology one. The tomographic result was consistent with the presumptive diagnosis in $103(34.92 \%)$ records. The correlation was not associated with any of the variables evaluated. $47(13.70 \%)$ of the records had not the presumptive diagnosis. Conclusion: There is high frequency of discordance between the tomographic results and presumptive diagnosis. One in ten tests does not have the presumptive diagnosis. Keywords: Diagnosis; scan; abdomen. (Source: MeSH NLM).

CITA SUGERIDA.

Goicochea Ruiz Virginia Nelly , Oyola García, Alfredo Enrique, Quispe llanzo Melisa Pamela, Muñoz Bendezú Zunilda Leyda. Concordancia entre el diagnóstico clínico presuntivo y el diagnóstico tomográfico

abdomino-pélvico. Rev méd panacea.2015; 5(2): 